\title{
EVALUACIÓN DEL RUIDO EN LA UNIDAD DE CUIDADOS INTENSIVOS NEONATAL ${ }^{1}$
}

\author{
EVALUATION OF NOISE IN THE NEONATAL INTENSIVE CARE UNIT \\ Alessandra Teixeira ${ }^{2}$, Flávia Andrade Fialho ${ }^{3}$, Iêda Maria Ávila Vargas ${ }^{4}$, Kátia Cristina de Souza Martins ${ }^{5}$, \\ Roberto Vale Machado ${ }^{6}$, Maria Efigênia Correia ${ }^{7}$
}

\section{RESUMEN}

Introducción: Con los avances en la tecnología y la investigación llevada a cabo en neonatología, ya es posible ver más allá de lo que podamos para salvaguardar las vidas de recién nacidos $(\mathrm{RN})$, proporcionando una calidad de vida. Para esto necesitamos tener una visión amplia que se ve más allá del individuo, el medio ambiente y el equipo que le rodea. Humanización debe impregnar todas las acciones tomadas en la Unidad de Cuidados Intensivos Neonatales (UCIN). Objetivo: En este contexto, el ruido ambiental emerge como un objeto de estudio de esta investigación tiene como objetivo identificar los niveles de ruido a que están expuestos los recién nacidos ingresados en una unidad específica de cuidados intensivos neonatales y discutirlos a la luz de las normas vigentes país. Materiales y Métodos: Teniendo en cuenta el tema de la parte delantera de esta investigación considera que es importante conocer la legislación que aborda este tema, específicamente el procedimiento estándar de Higiene Profesional Técnica (NHO 01) y la norma 15 (NR15). De acuerdo con la Norma 15 (NR 15) promulgado por el Ministerio de la Ordenanza $N^{\circ} 3214 / 1978$ del Trabajo, actualizado por el Decreto 203/2011, el ruido que se clasifica como el impacto del ruido o el ruido continuo también llamado intermitente, que se caracteriza los picos de energía acústica de menos de 1 (un) segundo a intervalos superiores a 1 (un) segundo. Resultados: Comenzamos la presentación de los resultados que describen el campo de estudio, los resultados de la investigación discutidos a la luz de las normas vigentes en el país. Discusión y Conclusiones: La institución por área de estudio es un centro de referencia para cuidados intensivos neonatales de la micro-región de Barbacena recibe pacientes de varios lugares. De acuerdo con la Resolución del Directorio $\mathrm{N}^{\circ} 7$ (RDC 07) se clasifica como un nivel de unidad de cuidados intensivos II(10). (Rev Cuid 2011; 2(2): 114-18)

Palabras clave: Enfermería, Ruído, Neonatología. (Fuente: DeCs BIREME).

\section{ABSTRACT}

Introduction: With advances in technology and research carried out in the nursery, it is possible to see beyond what we can to safeguard the lives of newborn (NB), providing a quality of life. For this we need to have a broad vision that looks beyond the individual, the environment and the team around him. Humanization must permeate all actions taken in the Neonatal Intensive Care Unit (NICU). Objective: In this context, environmental noise is emerging as an object of this research study aims to identify the noise levels they are exposed to the newly infants admitted to a specific unit and neonatal intensive care discussed in the light of existing rules the country. Materials and Methods: Given the theme of the front of this investigation considers it important to know the law that addresses this issue, specifically the standard procedure of Technical Vocational Hygiene (NHO 01) and Rule 15 (NR15). According with Rule 15 (NR 15) promulgated by the Ministry Order No. 3214/1978 of Labor, updated by Decree 203/2011, the noise impact is classified as noise or continuous noise also called intermittent, which features of acoustic energy peaks of less than 1 (one) second at intervals greater than 1 (one) second. Results: We begin the presentation of results that describe the field of study, research results discussed in the light of existing standards in the country. Discussion and Conclusions: The study area institution is a referral center for neonatal intensive care of the micro-region Barbacena receives patients from several places. According to Board Resolution No. 7 (RDC 07) is classified as a level II intensive care unit (10).

Key words: Nursing, Noise, Neonatology. (Source: DeCs BIREME).

\footnotetext{
${ }^{1}$ Artículo Original de Investigación.

${ }^{2,5,6}$ Enfermeiros, discentes do Curso de Especialização de Enfermagem em Terapia Intensiva Adulto e Neonato, da Faculdade de Enfermagem da Universidade Federal de Juiz de Fora. Pesquisadores do Núcleo de Pesquisa em Saúde Materno Infantil e Saúde Coletiva - FACENF - UFJF.

${ }^{3}$ Enfermeira, bolsista CAPES do Programa de Pós-Graduação Stricto Sensu Mestrado em Enfermagem da Escola de Enfermagem da Universidade Federal de Juiz de Fora (UFJF-MG) Brasil. Pesquisadora do Núcleo de Pesquisa em Saúde Materno Infantil e Saúde Coletiva - FACENF-UFJF.E-mail: flavinhafialho@bol.com.br

${ }^{4}$ Prof $^{a}$ Dr $^{a}$ da Faculdade de Enfermagem da Universidade Federal de Juiz de Fora-FACENF - UFJF - Líder do Núcleo de Pesquisa em Saúde Materno Infantil e Saúde Coletiva- FACENF - UFJF.

${ }^{7}$ Coordenadora do Curso de Especialização de Enfermagem em Terapia Intensiva Adulto e Neonato, da Faculdade de Enfermagem da Universidade Federal de Juiz de Fora. Pesquisadores do Núcleo de Pesquisa em Saúde Materno Infantil e Saúde Coletiva FACENF - UFJF.

Autor para Correspondencia: Flávia Andrade Fialho, Rua Santos Dumont, 128/303 Bairro: Centro Juiz de Fora/MG CEP: 36010-510 e-mail: flavinhafialho@bol.com.br Tel: (32) 3216-2299 


\section{INTRODUCCIÓN}

Con los avances en la tecnología y la investigación llevada a cabo en neonatología, ya es posible ver más allá de lo que podamos para salvaguardar las vidas de recién nacidos $(\mathrm{RN})$, proporcionando una calidad de vida. Para esto necesitamos tener una visión amplia que se ve más allá del individuo, el medio ambiente y el equipo que le rodea. Humanización debe impregnar todas las acciones tomadas en la Unidad de Cuidados Intensivos Neonatales (UCIN).

En este contexto, el ruido ambiental emerge como un objeto de estudio de esta investigación tiene como objetivo identificar los niveles de ruido a que están expuestos los recién nacidos ingresados en una unidad específica de cuidados intensivos neonatales y discutirlos a la luz de las normas vigentes país.

Para conceptualizar el ruido es conveniente aclarar lo que es el sonido, que se define como una perturbación de vibración producida en el órgano de la audición, en efecto, que las huelgas de la oreja, que se caracteriza por sensaciones de placer y agradable, mientras que el ruido está relacionado con desagradáveis sonidos(1). El ruido se utiliza para describir un sonido no deseado o molesto producido por las máquinas, equipos, bocinas y otros equipos y instrumentos(2).

El exceso de ruido en las Unidades de Cuidados Intensivos Neonatales (UCIN) los resultados de diversas fuentes, tales como la circulación de personas dentro de la unidad y el equipo de soporte de vida. Las condiciones ambientales afectan lo fisiológico y neurológico del recién nacido, por lo que el entorno físico no se pueden menospreciar, ya que ha demostrado ser una potente fuente de stress(3).

La Organización Internacional de ruido Consejo ha recomendado que los niveles de ruido en las áreas de cuidados intensivos no exceda de $45 \mathrm{~dB}$ (A) durante el día, $40 \mathrm{~dB}(\mathrm{~A})$ en la noche y $20 \mathrm{~dB}$ (A) durante la noche “(1).

No es permisible la exposición a niveles de ruido por encima de $115 \mathrm{~dB}$ (A) a las personas que no están adecuadamente protegidos. Este valor es perjudicial para la cóclea del oído humano, perjudicando a los de los adultos que están expuestos a estos ruidos durante sus horas de trabajo a los bebés que están expuestos al medio ambiente ruidoso durante días o meses, incluso durante su estancia en UTIN(4).

La exposición a ambientes ruidosos pueden provocar cambios fisiológicos, la disminución de la saturación de oxígeno $(\mathrm{O} 2)$, la frecuencia cardíaca y la presión intracraneal y los cambios en los patrones de sueño, puede llevar en casos extremos, incluso una pérdida auditiva(5).

El ruido en el entorno cerrado que es la UCIN contribuye a un mayor estrés de los profesionales del recién nacido y la salud que trabajan en esta unidad, en especial a la enfermera, que trabaja para un período más largo de esta unidad. El estrés de la unidad de recién nacidos causada por el ruido puede conducir a la irritabilidad y trastornos del sueño, la alteración de sus signos vitales y prevenir el desarrollo perfecto.

Mientras que en un ambiente libre de ruido de profesionales de la salud brindando atención de calidad y el recién nacido son capaces de desarrollarse adecuadamente. $\mathrm{Al}$ reflexionar sobre este tema hace que el estudio de la relevancia de la enfermería ha estado buscando a través de la construcción del conocimiento en la práctica el despliegue de medidas para minimizar los efectos nocivos de la hospitalización en la UCIN y garantizar la calidad de vida del recién nacido.

\section{MATERIALES Y MÉTODOS}

Teniendo en cuenta el tema de la parte delantera de esta investigación considera que es importante conocer la legislación que aborda este tema, específicamente el procedimiento estándar de Higiene Profesional Técnica (NHO 01) y la norma 15 (NR15).

De acuerdo con la Norma 15 (NR 15) promulgado por el Ministerio de la Ordenanza $N^{\circ} 3214 / 1978$ del Trabajo, actualizado por el Decreto 203/2011, el ruido que se clasifica como el impacto del ruido o el ruido continuo también llamado intermitente, que se caracteriza los picos de energía acústica de menos de 1 (un) segundo a intervalos superiores a 1 (un) segundo.

Esta norma establece que los niveles de ruido continuo o intermitente se mide en decibelios $(\mathrm{dB})$ con un instrumento operativo de nivel de presión acústica en el circuito de compensación "A" respuesta del circuito lento (SLOW). No es permisible la exposición a niveles de ruido por encima de $115 \mathrm{~dB}$ (A) a las personas que no están adecuadamente protegidos. El nivel de impacto también debe ser evaluado en decibelios $(\mathrm{dB})$ de funcionamiento del medidor de nivel de presión sonora en el circuito lineal y la respuesta del circuito a los impactos. La tolerancia de ruido de impacto es de 130 $\mathrm{dB}$ (lineal). En los intervalos entre los picos, el ruido existente debe ser evaluado como un ruido continuo.

En caso de indisponibilidad del sonido metros respuesta del circuito a presión a nivel del impacto, la lectura será válida en el circuito de respuesta rápida (FAST) y 
circuito de compensación " C". En este caso, el límite de tolerancia será de $120 \mathrm{~dB}(\mathrm{C})(6)$.

El 01 NHO tiene por objeto establecer los criterios y procedimientos para la evaluación de la exposición laboral al ruido, lo que implica un riesgo potencial. La norma se aplica a la exposición laboral al ruido y el impacto del ruido continuo o intermitente en cualquier situación de trabajo, pero no se centra en la caracterización de las condiciones de confort acústico. En virtud de esta norma la evaluación del ruido debe ser con el fin de caracterizar la exposición de los temas que ruídos(7).

El conjunto de mediciones deberán ser representativas de las condiciones reales de exposición ocupacional. Por lo tanto, la evaluación debe abarcar todas las condiciones, operativas y estándares ambientales.

Para las mediciones son representativos de la exposición a lo largo de la jornada de trabajo es importante que el período de muestreo está bien elegido. Si se identifican los ciclos repetitivos de la exposición durante el día, el muestreo debe incluir un número suficiente de ciclos. La muestra debe abarcar un mayor número de ciclos, si estos no son los niveles normales o presentan grandes variaciones en los valores.

Los procedimientos de evaluación deben interferir lo menos posible de las condiciones ambientales y las características operativas. No rutinarias las condiciones de exposición resultantes de las operaciones o procedimientos de trabajo predecible, pero poco comunes, como el mantenimiento preventivo debe ser evaluada e interpretada de manera aislada, teniendo en cuenta su contribución a la dosis diaria o nivel de exposición. En la caracterización de la exposición del sujeto al ruido se debe obtener de los datos administrativos con el apoyo de la observación de campo.

$\mathrm{Al}$ referirse a los procedimientos generales de la medición se puede mencionar que el equipo de medición cuando está en uso, deben ser calibrados y en perfecto estado electromecánico. Antes de comenzar las mediciones debe: verificar la integridad y la coherencia en la respuesta del instrumento electromecánico, verificar las condiciones de energía de la batería, ajuste los parámetros de medición, como el criterio a utilizar, la calibración de acuerdo con las instrucciones de el fabricante.

Antes de iniciar la medición de la exposición al ruido todos los involucrados deben ser informados y la medición no debe interferir con las actividades de costumbre, la rutina debe mantenerse, las medidas no afectan a la grabación de conversaciones. Debe ser invalidado e hizo nuevas medidas en la medida de la escala de patrones de carga fuera de los límites tolerados $\mathrm{DE} 1 \mathrm{~dB}$, el nivel de voltaje de la batería está por debajo del mínimo aceptable, no hay ningún daño a la integridad de los equipos electromecánicos.

Através de estos conceptos que nos proponemos llevar a cabo una investigación científica cuantitativa. La investigación científica es un procedimiento sistemático y racional que se da respuesta a problemas como los objetivos propuestos, y depone no es necesario cuando la información para responder a problema(8).

La investigación cuantitativa es un método que sea apropiado para el análisis estadístico de proceso de datos se debe aplicar cuando sea necesario es un estudio exploratorio de una situación particular o cuando se necesita un diagnóstico inicial(9).

En cuanto al procedimiento se puede decir que el estudio fue investigar el campo de la Unidad Neonatal de Cuidados Intensivos de un hospital público en la Zona da Mata de Minas. El estudio fue realizado por la autorización del administrador institución y la aprobación por el Comité de Ética, el dictamen 280/2010, siguiendo los pasos descritos a continuación:

1) Visita técnica al campo para identificar la capacidad de la unidad hospitalaria y el número de empleados que trabajan en la industria en este momento, también se observó la estructura física y las rutinas de las horas de visita.

2) Definir el lugar apropiado para la instalación de la unidad de campo audiodosímetro. El equipo se fijó en un sitio estratégico con el fin de grabar los sonidos a través de la unidad.

3) Instale la unidad de audiodosímetro.

4) El control diario de los niveles de ruido al que estaban expuestos los recién nacidos ingresados en la unidad. Esta supervisión se llevó a cabo mediante el registro de la información almacenada en la memoria del dispositivo, recogidos cada doce horas durante un período de veinticuatro horas en dos turnos, día y noche.

5) histogramas de impresión, el análisis estadístico de los datos y simplificación de las bases teóricas de los resultados.

6) Elaboración del informe final y la presentación de la misma a la institución y la comunidad científica. 
Tenga en cuenta que la instalación del dispositivo de impresión y el histograma fue acompañado por un trabajo técnico de seguridad debidamente capacitados.

\section{RESULTADOS Y DISCUSIÓN}

Comenzamos la presentación de los resultados que describen el campo de estudio, entonces, los resultados de la investigación discutidos a la luz de las normas vigentes en el país.La institución por área de estudio es un centro de referencia para cuidados intensivos neonatales de la micro-región de Barbacena recibe pacientes de varios lugares. De acuerdo con la Resolución del Directorio $\mathrm{N}^{\circ} 7$ (RDC 07) se clasifica como un nivel de unidad de cuidados intensivos II(10).

La estructura física de la unidad contiene un anti-sala utilizada para el resto del equipo, una corona, una limitación de espacio, dos baños separados por sexo para el equipo, una purga, tres salas, sala llamé, II y III. El estudio se llevó a cabo en la sala II, que mide $8,60 \times 4,15 \mathrm{~m}^{2}$ y cuenta con 06 camas distribuidas en forma de "C". La elección de esta sala se debió a un mayor número y más camas física neonatal ocupado en el momento de la encuesta.

El pabellón está diseñado principalmente para el cuidado de los recién nacidos prematuros clínicamente inestable. Trabaja en los servicios de emergencia, y el día comienza a las 7:00 am y terminan a las 19:00 horas, cuando se inicia el turno de la noche terminó a las 7:00 am del día siguiente.

Cada día, la unidad cuenta con dos pediatras, dos fisioterapeutas, dos residentes de la fisioterapia, dos enfermeras y un equipo de enfermería consta de seis auxiliares de enfermería. Aún circulan por la unidad de personal de limpieza, médicos de diversas especialidades, técnicos de Rx y los padres de los recién nacidos.

Refiriéndose a los resultados podemos decir que la intensidad del ruido se mide con un sonido de la marca Simpson 897 audiodosímetro dosímetro informe de analistas de la manufactura estadounidense, para descargar, calibrado y aprobado por el INMETRO. Ajustado circuito ponderado "A" respuesta lenta. Los rangos de niveles de presión sonora (NPS) se fijaron entre 50 y $130 \mathrm{~dB}$ a $5 \mathrm{~dB}$ como la tasa de compensación. El dispositivo se fija a una altura de un metro y $50 \mathrm{~cm}$ sobre el suelo a una distancia de $60 \mathrm{~cm}$ de la pared. Es de destacar que el dispositivo fue instalado en la UCIN dos días antes del comienzo de la recolección de datos en sí, para que los profesionales que trabajan allí se familiaricen con la presencia de los mismos.
La recolección de datos se llevó a cabo de manera efectiva en dos turnos de 24 horas en una fila, por un total de 48 horas durante un período de análisis de rutina de la unidad, en la que había una fiesta y no había ninguna fiesta oficial.

La unidad fue retirada de la sala a la realización de la impresión del histograma, siempre después de la aprobación de su deber. Después de imprimir el dispositivo fue reemplazado y se conecta a una nueva lectura.

El análisis de los registros muestran que durante el período de estudio de nivel equivalente (Leq), que representa el nivel promedio de ruido continuo y constante en $\mathrm{dB}(\mathrm{A})$, la sala de estudio fue inferior a $50 \mathrm{~dB}$ (A). Lo que se considera saludable, como la Asociación Brasileña de Normas Técnicas (ABNT) para establecer los niveles de ruido compatibles con el confort acústico para los seres humanos en diferentes entornos implica que los valores establecidos para las guarderías es de $35 \mathrm{~dB}$ (A) y $45 \mathrm{~dB}$ (A), de los cuales el valor de $35 \mathrm{~dB}$ (A) es deseable y 45 $\mathrm{dB}$ (A) es el aceptable(11).

Pero los registros muestran una variación significativa del nivel máximo (Lmax) que van desde 80,2 dB a 120.4dB. Es importante destacar que todos estos ruidos son de impacto, es decir, los picos de energía acústica tienen menos de un segundo de duración. Por lo tanto, está claro que los valores medidos fueron muy intensos y se acercó al valor de $130 \mathrm{~dB}$ (lineal), que es el límite reglamentario establecido por la norma NR-15.8

Esta norma establece que una persona sea expuesta a ruido de impacto de $130 \mathrm{~dB}$, se debe abandonar el lugar inmediatamente después de la exposición al ruido fuerte y sólo debe volver al sitio al día siguiente. Así, habrá un intervalo de tiempo, de modo que la audiencia para recuperar el impacto acústico sufrido. Lo que es imposible con un nacidos ingresados a la UCIN.

En base a estos, cabe mencionar que el campo de la enfermería de estudio a pesar de tener un Leq en el presente de ruido recomendados, se tenga en cuenta los picos de energía acústica, considerado el impacto del ruido que se producen principalmente por el equipo de alarma, la comunicación entre los profesionales de la industria y el incumplimiento de las rutinas en la industria, tales como puertas de mantener cerrada. Llegar a las variaciones entre $80.2 \mathrm{~dB}$ a $120,4 \mathrm{~dB}$, lo cual es preocupante, a considerar los efectos nocivos de la exposición al ruido para el recién nacido.

\section{CONCLUSIONES}

Al final de este estudio demuestran que el entorno físico 
de la unidad neonatal de cuidados intensivos no pueden ser ignoradas, lo que hace que la necesidad latente de un análisis más eficaz sobre el ruido que los recién nacidos están expuestos y los profesionales.

A pesar de la asistencia pre-término, estas unidades han pasado por grandes transformaciones, desde la perspectiva del cuidado del medio ambiente como el ruido y la luz, nos encontramos con que la UCIN estudiado expresado todavía en sus picos diarios de ruido, lo que representa un riesgo tanto para los recién nació en el hospital y para el equipo de salud y esto requiere de estrategias para su control y reducción.

Una posible estrategia sería la realización de actividades educativas con el fin de desarrollar y elevar el equipo para construir un modelo de atención dirigido a no sólo el cuidado humano, sino también las condiciones ambientales que afectan a los recién nacidos prematuros y de personal. Otra estrategia sería usar audiodosímetro sistemática de la unidad, un dispositivo que puede detectar el nivel de ruido, lo que impide un mayor daño a los recién nacidos.

Creemos que la inserción de la actividad de control de ruido que la excelencia en el proceso de rehabilitación y desarrollo del recién nacido y contribuir al bienestar colectivo, mientras que para deshacerse de la molestia del ruido, a menudo imperceptibles para el oído de adultos .

Es interesante que los profesionales están al tanto del alcance de estas cuestiones legislativas. Normas reglamentarias sobre la base de las acciones y los requisitos de puntos para la dirección de las instituciones de salud, para el bienestar del paciente. Por lo tanto, hay que destacar que la enfermera debe actuar con prudencia y discreción en los niveles de ruido no sólo benefician al paciente, sino también la autoridad sanitaria en el caso de auditorías técnicas y acreditaciones.

\section{REFERENCIAS BIBLIOGRÁFICAS}

1. Cheregatti, Aline Laurenti; Amorim, Carolina Padrão. Enfermagem Unidade de Terapia Intensiva. 1 ed. São Paulo: Martinari, 2010.

2. Carvalho, G.M. Enfermagem do Trabalho. EPU. Reempressão: São Paulo. 2001.

3. Scochi, Carmin Gracinda Silvan. et al. Cuidado Individualizado ao pequeno prematuro: o ambiente sensorial em unidade de terapia intensiva neonatal. Acta Paul Enf, v.14, n.1, Jan./ abr. , 2001.

4. Vieira, Sebastião Ivone. Manual de saúde e segurança do trabalho: Administração e gerenciamento de serviços. vol. 3, São Paulo: LTR, 2005.

5. Aurélio, F. S. Ruído em Unidade de Terapia Intensiva Neonatal. 2009. 118 f. Dissertação (Mestrado em Distúrbios da Comunicação Humana) Universidade Federal de Santa Maria.

6. Brasil. Ministério do Trabalho e Emprego. Secretaria de Inspeção do Trabalho. Norma Regulamentadora de Atividades e Operações Insalubres. Portaria 203 de 28 de janeiro de 2011. Brasília, 2011.

7. Brasil. Ministério do Trabalho e Emprego. Norma Regulamentadora de Higiene Ocupacional Procedimento Técnico. Avaliação da Exposição ao Ruído, Brasília, 2001.

8. Gil, Antônio Carlos, Como elaborar um projeto de Pesquisa. 3 ed. São Paulo: Atlas, 1996. 159 p.

9. Figueiredo, Nébia Maria Almeida de. Método e metodologia em pesquisa cientifica. $3^{\circ}$ ano, Yendis, 2009.

10. Brasil, Agência Nacional de Vigilância Sanitária, Resolução da Diretoria Colegiada - RDC No 7 de 24 de feve reiro de 2010. Brasília, 2010.

11. Associação Brasileira de Normas Técnicas - ABNT. Norma NBR 10152 - Níveis de Ruídos para conforto acústico, 1990. 